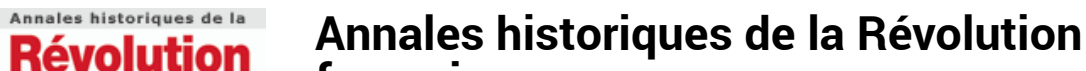

française française

356 | avril-juin 2009

Varia

\section{Gilbert Romme, Correspondance}

\section{Patrice Bret}

\section{OpenEdition}

\section{Journals}

Édition électronique

URL : https://journals.openedition.org/ahrf/10651

DOI : 10.4000/ahrf.10651

ISSN : 1952-403X

Éditeur :

Armand Colin, Société des études robespierristes

Édition imprimée

Date de publication : 1 avril 2009

Pagination : 219-221

ISBN : 978-2-200-92558-1

ISSN : 0003-4436

Référence électronique

Patrice Bret, "Gilbert Romme, Correspondance », Annales historiques de la Révolution française [En ligne], 356 | avril-juin 2009, mis en ligne le 17 décembre 2009, consulté le 23 avril 2022. URL : http:// journals.openedition.org/ahrf/10651; DOI : https://doi.org/10.4000/ahrf.10651

Ce document a été généré automatiquement le 23 avril 2022

Tous droits réservés 


\title{
Gilbert Romme, Correspondance
}

\author{
Patrice Bret
}

\section{RÉFÉRENCE}

Gilbert Romme, Correspondance,éd. Anne-Marie Bourdin, Philippe Bourdin, Jean Ehrard, Hélène Rol-Tanguy, Alexandre Tchoudinov, Clermont-Ferrand, Presses universitaires Blaise Pascal, 2006, vol. 1, 726 p. en 2 tomes (t. 1, 1774-1776 ; t. 2, 17771779), ISBN 2-84516-309-6, $32 €$.

1 L'édition critique de sources est toujours un événement qui mérite d'être salué, car leur mise à disposition des chercheurs est porteuse du développement de la recherche. Celle d'une correspondance l'est d'autant plus qu'elle échappe au caractère formel des documents officiels pour laisser transparaître la construction intime de l'identité de l'épistolier en fournissant des données irremplaçables pour la connaissance de sa vie et la compréhension de son itinéraire intellectuel. Inutile de préciser, en l'occurrence, que la correspondance des années de formation d'un futur révolutionnaire revêt une importance particulière pour les lecteurs de cette revue, auxquels il n'est pas nécessaire de présenter Charles-Gilbert Romme, l'un des "martyrs de Prairial ». Qu'il suffise de renvoyer à l'ouvrage de référence d'Alessandro Galante Garrone - à la mémoire duquel le présent recueil est dédié - publié à Turin en 1959 (trad. fr. : Gilbert Romme, histoire d'un révolutionnaire (1750-1795), Paris, Flammarion, 1971) et au colloque Gilbert Romme (17501795), tenu pour le bicentenaire de sa mort (Annales historiques de la Révolution française, $\mathrm{n}^{\circ} 304$, avril-juin 1996).

2 La correspondance en forme un utile complément, non seulement pour « préciser tel ou tel point de sa biographie, mais aussi pour embrasser plus concrètement la République des lettres depuis l'échelle européenne jusqu'à ses attendus provinciaux, depuis l'Ancien Régime jusqu'à son éradication ou sa transfiguration révolutionnaire " (Ph. Bourdin, p. 36). Pour ce faire, ont été exploités les fonds importants de Milan (Museo del Risorgimento), Saint-Pétersbourg (notamment à l'Institut d'histoire russe) et Moscou (notamment les Archives d'actes anciens de l'État russe), ceux de Paris (Bibliothèque 
nationale de France, NAF 4789-4790 ; Muséum national d'histoire naturelle), ClermontFerrand (Archives départementales, Bibliothèque communautaire et interuniversitaire) et Riom (Archives communales), sans compter ceux de Strasbourg (Archives départementales et communales, Bibliothèque nationale et universitaire), des bibliothèques de Genève, Berlin et Zurich ou d'archives privées. Dans l'annexe II qui détaille ces sources (p. 79-92) ne figurent étonnamment ni les archives des assemblées et du pouvoir exécutif aux Archives nationales, ni les documents de la Bibliothèque municipale de Riom (AG 65-67, AG 1-83) signalés par Les archives des scientifiques, XVI ${ }^{e}$ $X X^{e}$. siècle Guide des fonds conservés en France (éd. Thérèse Charmasson, Paris, Ed. du CTHS, 2008, p. 515-516), ni ceux de l'Académie des sciences (voir Euvres de Lavoisier, Correspondance, vol. 7, 1792-1794, Paris, Académie des sciences et Ed. Hermann, sous presse). Saluons d'abord la qualité d'ensemble de cette édition soignée, qui frappe aussitôt le lecteur par la rigueur - les règles d'édition données dans la préface ont été définies avec l'aide de Georges Dulac - et le soin apporté à sa réalisation. Sous la houlette savante de Jean Ehrard, l'équipe éditoriale, solidement constituée autour de l'université Blaise Pascal - la présence d'Alexandre Tchoudinov garantissant le traitement du versant russe de la carrière de Romme - a eu ponctuellement recours à une trentaine de spécialistes, notamment du groupe d'Alembert et de la Revue Diderot et l'Encyclopédie.

3 Le contexte général est fermement tracé par la présentation de Philippe Bourdin sur «Romme dans l'historiographie de la Révolution française» (p. 9-37) et par l'introduction de Jean Ehrard au premier volume, « un étudiant riomois à Paris » (p. 41-73), qui résume et donne la grille de lecture d'un ensemble de 136 lettres particulièrement vivantes, dont l'apparat critique, riche et efficace, ne se limite pas aux notes qui accompagnent chacune d'elles. L'édition du corpus est en effet précédée de cinq « annexes» (p. 75-114) - une biographie synthétique, les sources, les domiciles parisiens de Romme, les 77 ouvrages (surtout scientifiques) ou journaux cités ou évoqués dans la correspondance, avec renvoi aux lettres de la période, et la poste au XVIII ${ }^{e}$ siècle- et suivie de cinq "appendices » (p. 589-631) présentant des attestations d'études de Romme, l'inventaire de son cabinet de physique, ses remarques sur la traduction de l'Essai philosophique concernant l'entendement humain de Locke par Coste et de nombreuses notes de lectures et anecdotes recueillies par Romme. Suivent encore d'utiles notices biographiques sur les protagonistes de la correspondance (p. 635-686). Face à un tel foisonnement, les petites lacunes ou scories inhérentes à une telle entreprise, au demeurant fort rares, ne méritent pas d'être relevées - p. 339, n. 5, le botaniste Danbey est Joseph Dombey (1742-1794). Était-il nécessaire dans l'index d'affubler le mathématicien Gaspard Monge du titre de comte de Péluse porté trente ans plus tard?

4 Dans le Paris des Lumières, le jeune provincial Romme découvre la vie intellectuelle de la capitale auprès du naturaliste Guettard et du poète Delille et tisse un réseau de relations dont il envoie, en décembre 1778, la liste à son ami Gabriel Dubreul, directeur de la poste aux lettres, en distinguant ceux de la « haute société ", à commencer par le comte Golovkine, et les "savans et ecrivains », en tête desquels figure l'abbé Rozier, rédacteur du Journal de physique (p. 462-463). En donnant des cours particuliers de mathématiques, Romme découvre en même temps les contrastes sociaux avant de se fixer tour à tour chez ce dernier, auprès de la comtesse d'Harville puis à l'Hôtel Stroganov. Médecin éclairé resté à Riom, le docteur Antoine Boirat joue le rôle de mentor. Il semble projeter sur le jeune homme ses propres ambitions refoulées - 
bientôt reçu correspondant de la Société royale de médecine -, mais il le conseille avec une grande lucidité politique et sociale pour mener une carrière scientifique, à l'instar du frère aîné, Charles Romme, professeur d'hydrographie et mathématicien, correspondant de l'Académie royale des sciences en août 1778 et membre du réseau d'observateurs de météorologie pour Lavoisier. Si ce dernier n'apparaît guère dans la correspondance, les échanges font apparaître la vitalité des liens entre la capitale et la province, où Romme envisageait de revenir. Affligé par la disgrâce de Turgot et de la raison réformatrice, mais rallié à l'intégrité de Necker, il délaisse finalement la médecine, après avoir suivi avec assiduité les cours d'anatomie et de chimie, pour se consacrer aux mathématiques, jusqu'à son passage en Russie, en août 1779, comme gouverneur du comte Paul Stroganov. Quelques mois plus tôt, il écrit: "Je déteste l'histoire presque autant que la simple littérature », sauf l'histoire des arts et des sciences seule "vraiment utile essentielle ", à la différence de l'histoire politique, «celle des carnage[s], des injustices, de la cruauté, de l'ambition des hommes de tous les ages [...] À mes yeux, un héros est toujours un fou et trop souvent un coquin qu'on décore parce qu'on le craint » (p. 497). Sans doute ne fut-il ni l'un ni l'autre. La suite de cette correspondance est attendue avec impatience. À terme, elle viendra préciser l'itinéraire intellectuel de Romme au contact de l'étranger et au retour en France, souligner son action entre le politique et le savant, comme représentant du peuple et comme membre du Comité d'instruction publique - notamment dans le calendrier républicain et plus généralement dans les poids et mesures - et probablement renouveler encore notre vision de son itinéraire révolutionnaire. 